
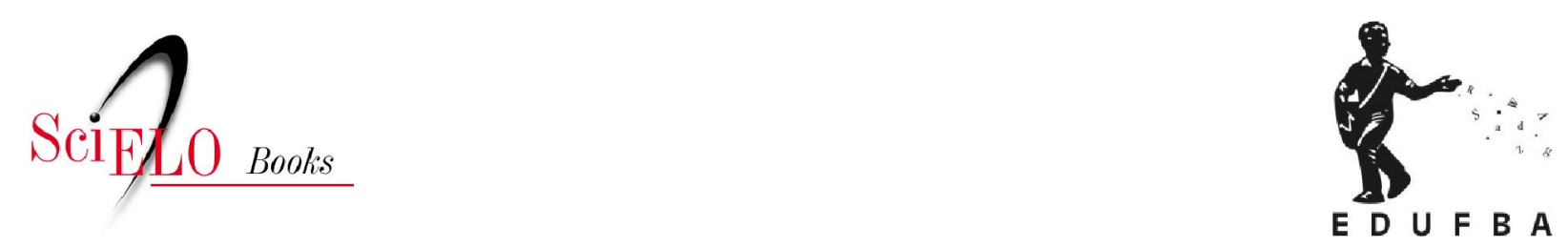

\title{
Transparência governamental eletrônica para Accountability
}

\author{
Maria Alexandra Cunha \\ Marco Antônio Carvalho Teixeira \\ Taiane Ritta Coelho \\ Maria Camila Florêncio da Silva \\ Stefania Lapolla Cantoni
}

\section{SciELO Books / SciELO Livros / SciELO Libros}

CUNHA, M.A., TEIXEIRA, M.A.C., COELHO, T.R., SILVA, M.C.F., and CANTONI, S.L.

Transparência governamental eletrônica para Accountability. In: PINHO, J.A.G., ed. Artefatos digitais para mobilização da sociedade civil: perspectivas para avanço da democracia [online]. Salvador:

EDUFBA, 2016, pp. 119-139. ISBN: 978-85-232-1877-5.

https://doi.org/10.7476/9788523218775.0007.

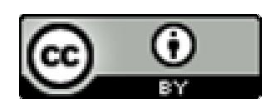

All the contents of this work, except where otherwise noted, is licensed under a Creative Commons Attribution $\underline{4.0 \text { International license. }}$

Todo o conteúdo deste trabalho, exceto quando houver ressalva, é publicado sob a licença Creative Commons Atribição 4.0.

Todo el contenido de esta obra, excepto donde se indique lo contrario, está bajo licencia de la licencia $\underline{\text { Creative }}$ Commons Reconocimento 4.0. 


\section{TRANSPARÊNCIA GOVERNAMENTAL ELETRÔNICA PARA ACCOUNTABILITY}

MARIA ALEXANDRA CUNHA, MARCO ANTÔNIO CARVALHO TEIXEIRA, TAIANE RITTA COELHO, MARIA CAMILA FLORÊNCIO DA SILVA, STEFANIA LAPOLLA CANTONI

INTRODUÇÃO

A Tecnologia da Informação e Comunicação (TIC) tem sido apontada como potencializadora da transparência e accountabilty tanto pela literatura internacional como pela produção nacional. Neste texto, utilizamos a literatura e casos práticos para argumentar que o uso das TIC para transparência faz sentido quando leva à melhoria da accountability. Para construir a nossa argumentação, partimos da definição de "transparência" e "accountability" e investigamos como, nos textos de transparência, a accountability é referenciada e vice-versa, como aqueles que escrevem sobre accountability fazem uso do termo "transparência”. É certo que há um razoável entendimento de que os processos de transparência governamental podem ser melhorados com a utilização da TIC, mas não há consenso sobre isso. Na segunda parte do capítulo, as visões otimista e pessimista sobre a transparência refletem-se também no uso de tecnologia. Descrevemos as diferentes dimensões utilizadas para avaliar transparência em sites governamentais. Finalmente, a partir de casos práticos, problematizamos transparência e accountability e sugerimos 
que o uso das TIC para a transparência é importante quando conduz a melhoria dos mecanismos de accountability.

\section{TRANSPARÊNCIA E ACCOUNTABILITY}

A transparência e o direito de acesso à informação governamental são internacionalmente considerados como essenciais para várias funções da democracia, como participação e accountability. (BERTOT; JAEGER; GRIMES, 2012) De acordo com as Nações Unidas, os conceitos de integridade, transparência e accountability são reconhecidos pela maioria dos países membros como fundamentos essenciais no setor público. (ARMSTRONG, E., 2005)

Vários acadêmicos examinaram maneiras de como a internet mudou a comunicação entre governos e seus constituintes locais. (ARMSTRONG, C., 2011; BERTOT; JAEGER; GRIMES, 2012; GRIMMELIKHUIJSEN, 2009) Estes autores têm sugerido que quanto mais informações os governos disponibilizam, a confiança e a eficiência do governo tendem a ser mais positivas. A transparência governamental é importante (ARMSTRONG, C., 2011; BERTOT; JAEGER; GRIMES, 2012; MEIJER, 2003), mas não é de fácil abordagem, devido ao "nevoeiro" que envolve o conceito. Para Liem (2007), existe uma falta de definição do termo transparência, de um conceito comum, o que leva por vezes a análises realizadas em um nível bastante abstrato. A transparência é vista por vários autores como conceito amplo e de difícil descrição. (GRIMMELIKHUIJSEN, 2009; LOURENÇO et al., 2013; MICHENER; BERSCH, 2013; RELLY; SABHARWAL, 2009)

O Quadro 1 resume diversos conceitos de transparência encontrados na literatura. Pode-se tratar transparência como uma relação horizontal entre o governo e o cidadão, permitindo o que é melhor para todos. (GRIMMELIKHUIJSEN, 2009) Alguns autores associam transparência ao acesso ou à disponibilidade de informação. (ARMSTRONG, C., 2011; CERRILLO-I-MARTÍNEZ, 2011; CRUZ; ÁLVARO, 2012; DEL SOL, 
2013; GRIMMELIKHUIJSEN, 2012; GRIMMELIKHUIJSEN et al., 2013; HALACHMI; GREILING, 2013; MEIJER; BANNISTER; GRIMMELIKHUIJSEN, 2011; PARAJULI, 2007; RIBEIRO; MATHEUS; VAZ, 2011; WELCH, 2012) Associam-na também à abertura das informações governamentais ao público externo ou à disponibilidade gratuita da informação.

Há outros autores que percebem transparência quando ocorre a possibilidade de controle ou monitoramento das ações públicas por parte dos cidadãos. (DAWES, 2010; GRIMMELIKHUIJSEN et al., 2013; GRIMMELIKHUIJSEN; WELCH, 2012; HALACHMI; GREILING, 2013; JACQUES; QUINTANA; MACAGNAN, 2013; MEIJER; BANNISTER; GRIMMELIKHUIJSEN, 2011) Assim, a transparência acontece à medida em que uma entidade revela informações relevantes sobre o seu próprio processo decisório, procedimentos, funcionamento e desempenho, capazes de subsidiar o processo decisório do cidadão. (GRIMMELIKHUIJSEN et al., 2013; JACQUES; QUINTANA; MACAGNAN, 2013)

Quadro 1 - Conceitos encontrados na literatura - Transparência

\begin{tabular}{|c|c|c|}
\hline ATRIBUTO & CONCEITO & REFERÊNCIAS \\
\hline \multirow{4}{*}{$\begin{array}{l}\text { Como um } \\
\text { conceito Amplo }\end{array}$} & $\begin{array}{l}\text { "transparência" oferece um conceito } \\
\text { de "bem ambivalente", com uma } \\
\text { carga normativa positiva. }\end{array}$ & $\begin{array}{l}\text { Michener e Bersch } \\
(2013)\end{array}$ \\
\hline & $\begin{array}{l}\text { Transparência é visto por políticos } \\
\text { e alguns estudiosos como uma } \\
\text { panaceia para permitir "boa } \\
\text { governação". }\end{array}$ & $\begin{array}{l}\text { Grimmelikhuijsen } \\
(2009)\end{array}$ \\
\hline & $\begin{array}{l}\text { A transparência é um conceito amplo } \\
\text { [...] "A transparência é a condução dos } \\
\text { assuntos públicos ao ar livre ou está } \\
\text { sujeito a escrutínio público.". }\end{array}$ & $\begin{array}{l}\text { Relly e Sabharwal } \\
(2009)\end{array}$ \\
\hline & $\begin{array}{l}\text { Transparência é um termo complexo, } \\
\text { que pode ser analisado por meio de } \\
\text { um conjunto de dicotomias, sendo } \\
\text { principal aquela que ocorre entre o } \\
\text { evento e o processo de transparência. }\end{array}$ & Lourenço et al. (2013) \\
\hline
\end{tabular}




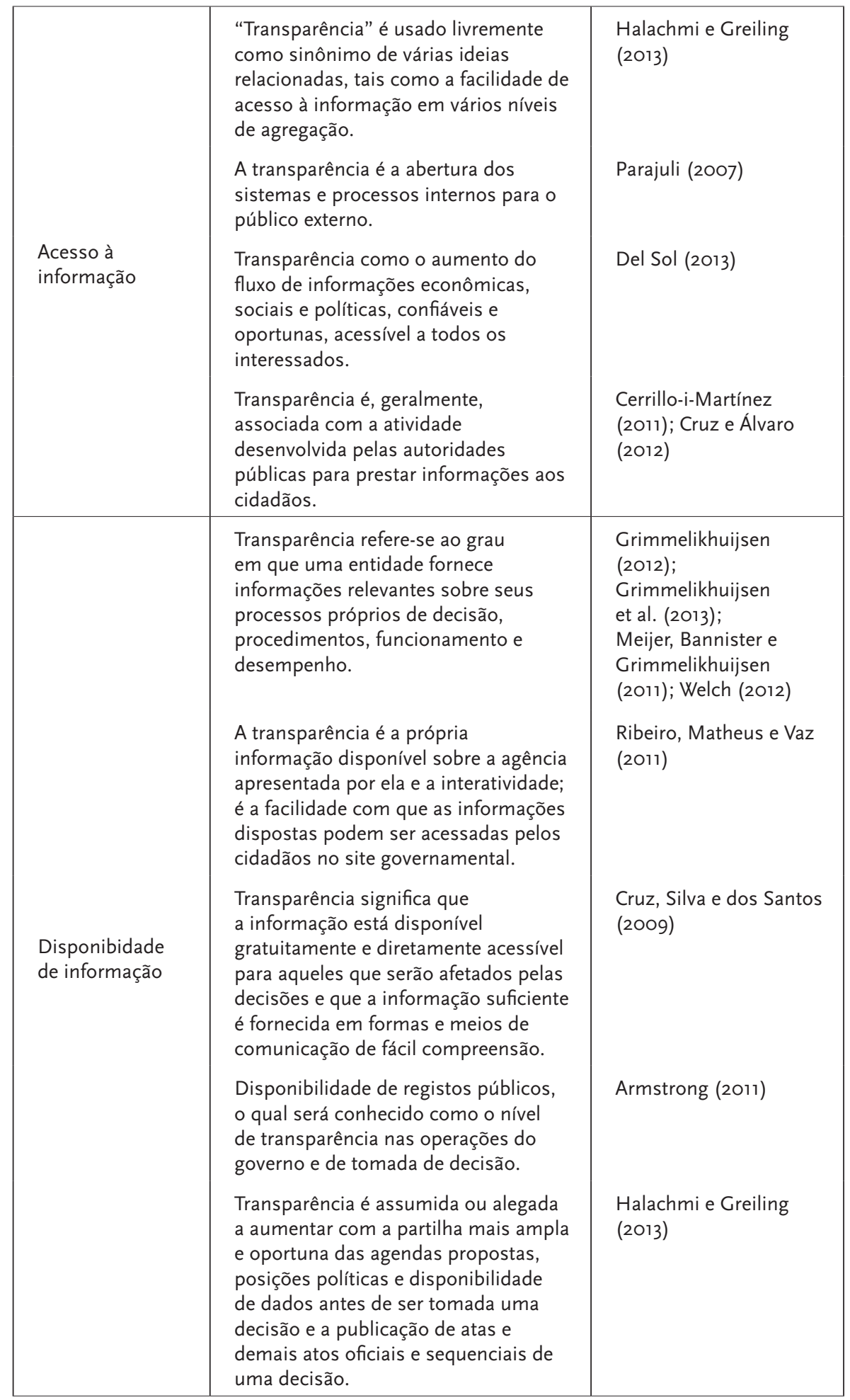




\begin{tabular}{|c|c|c|}
\hline & $\begin{array}{l}\text { Transparência pode ser direcionada } \\
\text { para fora (governo observando } \\
\text { cidadãos), para dentro (cidadãos } \\
\text { observando governo), para cima } \\
\text { (subordinados observando } \\
\text { seus supervisores) e para baixo } \\
\text { (supervisores observando } \\
\text { subordinados), ou qualquer } \\
\text { combinação desses. }\end{array}$ & $\begin{array}{l}\text { Meijer, Bannister e } \\
\text { Grimmelikhuijsen } \\
(2011)\end{array}$ \\
\hline & $\begin{array}{l}\text { Existe transparência quando os } \\
\text { cidadãos têm mais controle e formas } \\
\text { alternativas de acesso aos dados } \\
\text { brutos e influenciam o seu nível de } \\
\text { agregação. }\end{array}$ & $\begin{array}{l}\text { Halachmi e Greiling } \\
(2013)\end{array}$ \\
\hline & $\begin{array}{l}\text { Transparência à luz das tendências } \\
\text { sociais mais amplas sobre a } \\
\text { legitimidade, confiança e abertura. } \\
\text { A transparência definida como a falta } \\
\text { de sigilo e uma abertura ao escrutínio } \\
\text { público - é tradicionalmente } \\
\text { considerada como um meio para } \\
\text { reduzir a incerteza e aumentar a } \\
\text { confiança do público. }\end{array}$ & Dawes (2010) \\
\hline \multirow[t]{3}{*}{$\begin{array}{l}\text { Disponibilidade } \\
\text { e controle pelo } \\
\text { cidadão }\end{array}$} & $\begin{array}{l}\text { Transparência é vista como um } \\
\text { instrumento de política de governo: } \\
\text { pelo fornecimento de informações, o } \\
\text { governo facilita a tomada de decisão } \\
\text { dos cidadãos. Em segundo lugar, } \\
\text { o termo transparência é dito como } \\
\text { sendo um valor democrático. }\end{array}$ & $\begin{array}{l}\text { Grimmelikhuijsen e } \\
\text { Welch (2012) }\end{array}$ \\
\hline & $\begin{array}{l}\text { A transparência, mesmo derivando } \\
\text { diretamente do princípio } \\
\text { constitucional da publicidade, num } \\
\text { conceito mais amplo, abrange } \\
\text { a evidenciação de informações } \\
\text { confiáveis, relevantes, oportunas e } \\
\text { compreensíveis, capazes de subsidiar } \\
\text { o processo de controle social pela } \\
\text { sociedade. Transparência requer mais } \\
\text { do que se apresentar publicamente } \\
\text { ou ser visto por um número cada vez } \\
\text { maior de pessoas. }\end{array}$ & $\begin{array}{l}\text { Jacques, Quintana e } \\
\text { Macagnan (2013) }\end{array}$ \\
\hline & $\begin{array}{l}\text { O processo de transparência ocorre } \\
\text { "quando as organizações promovem } \\
\text { a tomada de decisão visível, éaberto } \\
\text { para a sugestão do público, permite } \\
\text { que o público escolha o máximo de } \\
\text { serviços e trabalha em cooperação } \\
\text { com outras organizações para fins } \\
\text { públicos comuns. }\end{array}$ & Armstrong (2011) \\
\hline
\end{tabular}




\begin{tabular}{|c|c|c|}
\hline $\begin{array}{l}\text { Disponibilidade } \\
\text { e monitoramento }\end{array}$ & $\begin{array}{l}\text { Transparência é a disponibilidade de } \\
\text { informações sobre uma organização } \\
\text { ou ator que permite que agentes } \\
\text { externos monitorem o funcionamento } \\
\text { interno, a realização dessa } \\
\text { organização ou ator. }\end{array}$ & $\begin{array}{l}\text { Grimmelikhuijsen } \\
\text { (2012); } \\
\text { Grimmelikhuijsen } \\
\text { e Welch (2012); } \\
\text { Grimmelikhuijsen } \\
\text { et al. (2013); } \\
\text { Meijer, Bannister e } \\
\text { Grimmelikhuijsen } \\
\text { (2011); Welch (2012), } \\
\text { p18, p22 } \\
\text { Grimmelikhuijsen et } \\
\text { al. (2013); Bannister } \\
\text { e Grimmelikhuijsen } \\
\text { (2011) }\end{array}$ \\
\hline $\begin{array}{l}\text { Quanto à } \\
\text { indisponibilidade } \\
\text { de transparência }\end{array}$ & $\begin{array}{l}\text { Ocorre em casos excepcionais em que } \\
\text { se justifica por segredo de Estado ou } \\
\text { por segurança da própria sociedade. } \\
\text { Todavia, deve ser em caráter de } \\
\text { exceção e não como regra. Passado } \\
\text { o perigo, a sociedade deve tomar } \\
\text { conhecimento do que ocorreu. }\end{array}$ & Bobbio (2000) \\
\hline Accountability & $\begin{array}{l}\text { Os termos accountability e } \\
\text { transparência estão estreitamente } \\
\text { relacionados. A diminuição da } \\
\text { opacidade administrativa propicia } \\
\text { maior possibilidade de conhecimento } \\
\text { do cidadão sobre a res publica. } \\
\text { Transparência concentra-se em } \\
\text { accountability. Seja qual for o caso, a } \\
\text { transparência é sempre uma condição } \\
\text { necessária como o primeiro passo no } \\
\text { processo de prestação de contas. }\end{array}$ & $\begin{array}{l}\text { Del Sol (2013); } \\
\text { Grimmelikhuijsen } \\
\text { (2009); Halachmi } \\
\text { e Greiling (2013); } \\
\text { Meijer, Bannister e } \\
\text { Grimmelikhuijsen } \\
(2011)\end{array}$ \\
\hline
\end{tabular}

Também foram encontrados autores (por exemplo, ALVES; SOUZA, 2011; CRUZ; ÁLVARO, 2012; CRUZ; SILVA; SANTOS, 2009; FRANCO et al., 2014) que abordam em seus textos a transparência fiscal, ou seja, a "possibilidade do acompanhamento claro, transparente, da execução orçamentária e das finanças públicas” (CRUZ; ÁLVARO, 2012), muitas vezes se reportando à Lei de Responsabilidade Fiscal.

A partir do conjunto dos autores citados, delimita-se o conceito. Assim, neste capítulo, transparência refere-se ao grau de disponibilidade de informação sobre uma agência, que pode ser acessada por 
todos os cidadãos e que permita que estes possam controlar e monitorar o funcionamento desta agência.

Alguns autores associam transparência à accountability (por exemplo, BAIRRAL; SILVA, 2013; CHEN, 2012; DEL SOL, 2013; GRIMMELIKHUIJSEN, 2012; LOUREIRO; TEIXEIRA; PRADO, 2008; LOURENÇO et al., 2013; MARENGO; DIEHL, 2011; MEIJER, 2003; RAUPP; PINHO, 2011; RIBEIRO; MATHEUS; VAZ, 2011), sendo então accountability definida como a obrigação de os funcionários públicos informarem sobre o uso dos recursos públicos e responsabilização do governo ao público para atender aos objetivos de desempenho declarados. (ARMSTRONG, E., 2005; BEHN, 2001; BOVENS, 2007; WONG; WELCH, 2004 apud LOURENÇO et al., 2013) A “[...] responsabilidade democrática exige que os governos aumentem a transparência, divulgando mais informações para os cidadãos, promovendo o controle das despesas públicas e prevenção da corrupção e desperdício de recursos públicos". (PINHO, 2008, p. 478) No entanto, “[...] a transparência é uma condição necessária, mas não suficiente, para assegurar uma maior accountability". (DEL SOL, 2013; HALACHMI; GREILING, 2013)

Em 1990, Campos com o seu emblemático texto "Accountability: quando poderemos traduzi-la para o português?”, concluiu que a inexistência de palavra que traduza seu significado no dicionário português se dá pelo aspecto cultural da Administração Pública não ser accountable. Quase 20 anos depois, Pinho e Sacramento (2009) escrevem o texto "Accountability: já podemos traduzi-la para o português?" onde concluem que apesar dos avanços, ainda estamos muito longe de construir uma verdadeira cultura de accountability. De acordo com Medeiros, Crantschaninov e Silva (2013, p. 1344), o termo que ganhou força após a queda do regime militar “[...] circunda a literatura em companhia de expressões como controle social, participação e a própria democratização do Estado" e se mistura com conceitos e abordagens relacionadas a perspectivas e visões, nem sempre de uma forma correta. (ROCHA, 2011) Nem sempre, aliás, tais conceitos e abordagens ficam evidentes. 
Com o fim de investigar a relação entre transparência e accountability, mais especificamente se transparência é elemento fundante deste, foi realizado um levantamento e análise da literatura nacional sobre accountability nos periódicos brasileiros classificados pela Comissão de Aperfeiçoamento de Pessoal do Nível Superior (Capes) como A2, B1 e B2 em Administração. O que o levantamento evidenciou foi que dos 38 (trinta e oito artigos), 11 (onze) não conceituavam accountability. Dentre os demais 27 (vinte e sete), 13 (treze) pressupunham transparência como sendo parte de accountability, e os outros 14 (quatorze) se dividiam entre 9 (nove) que não pressupunham e 5 (cinco) em que não foi possível ter certeza justamente por utilizar expressões como “acesso à informação", sem se referir ao termo. Disso se extrai que apenas $1 / 3$ dos trabalhos pressupõem que transparência é um elemento de accountability. Nestes, "[...] a transparência trata da visibilidade dos atos praticados no setor público e evidencia o acesso às informações e sua compreensão". (FONTES FILHO, NAVES, 2014, p. 9) Neste sentido, poderia ser considerada como "[...] a mais importante categoria da accountability como instrumento para avaliar o desempenho organizacional. Um requisito-chave para todas as outras. A transparência visa a mostrar a atuação das organizações e dos agentes públicos" (ROCHA, 2013, p. 72), "[...] uma condição sine qua non para o funcionamento dos mecanismos de responsabilização, já que, de fato, esses mecanismos de controle e fiscalização são fortemente condicionados pela transparência e pela visibilidade das ações do poder público". (CENEVIVA, FARAH, 2012, p. 1000) Nos outros 2/3 dos trabalhos, a definição aparece vinculada à responsabilização ou prestação de contas e apenas 10 (dez) dos 38 (trinta e oito) definem o que é transparência. Isso não quer dizer, contudo, que o termo não se fizesse presente, pois, na maior parte deles, "transparência" é empregada como uma qualidade da administração ou sinônimo de acesso à informação.

Também investigou-se como accountability aparece nos textos de transparência. Como observado por Grimmelikhuijsen (2012), a transparência, que visa melhorar a accountability permite que terceiros acessem e controlem as ações do governo. A informação disponível, 
por sua vez, capacita os cidadãos e, portanto, torna o governo mais democrático e mais confiável. (GRIMMELIKHUIJSEN, 2012; MEIJER, 2009; PINHO; SACRAMENTO, 2004; RIBEIRO; MATHEUS; VAZ, 2011) De acordo com Raupp e Pinho (2011),

[...] a transparência governamental é uma condição sine qua non para o funcionamento dos mecanismos de responsabilização, já que, de fato, esses mecanismos de controle e fiscalização são extremamente condicionados pela transparência e pela visibilidade das ações do poder público.

Neste sentido, a responsabilidade democrática exige que os governos aumentem a transparência, divulgando mais informações para os cidadãos, para que eles possam controlar as ações dos agentes públicos. (LOURENÇO et al., 2013)

Apesar da relação entre os conceitos, transparência e accountability não são sinônimos. (RIBEIRO; MATHEUS; VAZ, 2011) Deve notar-se que a transparência é uma condição necessária, mas não suficiente, para assegurar uma maior responsabilização pública (HALACHMI; GREILING, 2013), sendo o primeiro passo no processo de accountability. (MEIJER, 2003)

\section{O PAPEL DA TECNOLOGIA PARA A TRANSPARÊNCIA}

As Tecnologias de Informação e Comunicação (TIC) oferecem novas maneiras de aumentar a transparência no governo. (HALACHMI; GREILING, 2013) As TIC permitem que governos armazenem e divulguem grandes quantidades de dados a um custo baixo (MEIJER, 2007, 2009), dando possibilidades aos cidadãos de inspecionar o que as agências estão fazendo quase em tempo real. Os websites são uma ferramenta importante para a transparência, permitindo que as organizações governamentais forneçam as informações pró-ativamente. (MEIJER, 2011) E as mídias sociais têm tido um efeito transformador sobre as maneiras pelas quais as pessoas interagem umas com as outras e com os governos, bem como nas maneiras pelas quais os governos podem promover a transparência. (BERTOT; JAEGER; GRIMES, 2012) 
A transparência mediada pelas TIC visa melhorar a prestação de contas públicas, permite que terceiros acessem e fiscalizem as ações do governo. A melhor informação, por sua vez, capacita os cidadãos e, portanto, leva a governos mais democráticos e mais confiáveis. (GRIMMELIKHUIJSEN, 2012; MEIJER, 2009) Desta forma, o acesso à informação pode diminuir a assimetria entre a sociedade e o governo. (ANGÉLICO; ANTONIO, TEIXEIRA, 2012)

Quanto aos efeitos que a transparência pode trazer para a administração pública, pode-se dizer que há, em maior número, uma visão otimista, mas há registros de uma outra, pessimista. Embora a maioria dos autores reconheça que há muitos efeitos positivos de transparência, alguns outros chamam a atenção para alguns aspectos negativos.

Os "otimistas" abordam que uma maior transparência, que resulta de uma melhor utilização das TIC pode aumentar a confiança dos cidadãos, pode servir para limitar ou prevenir muitas oportunidades de comportamento corrupto (BERTOT; JAEGER; GRIMES, 2012), é um componente importante para a boa governança e pode aumentar a eficiência dos recursos, é essencial para a participação democrática, para precisão das informações do governo, e o fornecimento de informações para os públicos, empresas e jornalistas, entre outras funções essenciais na sociedade. (CULLIER; PIOTROWSKI, 2009; HOLMSTRÖM, 1979; MULGAN, 2007; QUINN, 2003; REYLEA, 2009; SHULER; JAEGER; BERTOT, 2010) Niklas Luhmann (2000) já declarou que a transparência é um primeiro passo necessário para restaurar a confiança dos cidadãos no governo. Ela cria uma cultura de abertura e isso proporciona efeitos positivos de confiança. (MEIJER; BANNISTER; GRIMMELIKHUIJSEN, 2011) A transparência aumenta a exposição das operações do governo ao escrutínio dos vários componentes do sistema político (MOON, 2002; TOLBERT; MOSSBERGER, 2006), aumentando o risco de ser pego. (DEL SOL, 2013) Os decisores políticos e estudiosos veem a transparência como um facilitador da boa governança (HOOD, 2006) porque ela estimula um melhor desempenho e aumenta a eficiência na alocação dos recursos. (DEL SOL, 2013; MEIJER, 2009) A transparência tornase uma ferramenta necessária para monitorar e avaliar o desempenho 
dos representantes e servidores públicos. Além disso, é um componente importante da boa governação e da qualidade institucional. (ACEMOGLU; JOHNSON; ROBINSON, 2002; KAUFMAN; KRAAY, 2002; WILLIAMSON, 2000) A transparência e o direito de acesso à informação do governo são considerados essenciais para a participação democrática. (BERTOT; JAEGER; GRIMES, 2010) Isto significa que, por meio do melhor entendimento das operações do governo que vem de uma maior transparência, os cidadãos poderão ter poderes para ter uma maior capacidade de influenciar as prioridades e procedimentos operacionais do governo. (HALACHMI; GREILING, 2013)

Por outro lado, os "pessimistas" assumem uma postura mais crítica e dizem que a transparência pode ocasionar a redução na eficiência administrativa e retardar as operações do governo devido à reduzida capacidade operacional que resulta do consumo de recursos escassos, aumentando os custos. (AIKINS; KRANE, 2012; HALACHMI; GREILING, 2013; LIEM, 2007) Ela pode ainda, comprometer "prestação de contas", facilitando o jogo político e pode reduzir a legitimidade de um determinado governo. (BOVENS, 2007; HALACHMI; GREILING, 2013) Liem (2007) observa que "a maior objeção contra a transparência é que ela pode ocasionar a redução na eficiência administrativa." Por exemplo, a transparência pode fazer tornar algumas ações administrativas mais eficazes, porém os mesmos esforços podem aumentar o custo marginal das operações do governo, principalmente quando há implicações nos recursos que são consumidos para alcançar a transparência. (HALACHMI; GREILING, 2013) Desta forma, a disponibilidade de informações poderiam tornar os procedimentos mais burocráticos em vez de aproximá-los dos cidadãos. (LIEM, 2007) Para Bovens (2005, p. 195), “[...] não há uma relação absoluta proporcional entre transparência e legitimidade”. Ainda segundo o autor, o aumento da transparência pode comprometer a accountability. O excesso de informação ou de muitos detalhes pode ser usado deliberadamente para intimidar e cercear o escrutínio público e a revelação de escândalos ou de algo que pode sugerir sua possível existência, pode ter o objetivo de reduzir a legitimidade de um determinado governo ou um arranjo 
de governança. (HALACHMI; GREILING, 2013) A transparência pode levar à desmistificação do governo, convidando a desafios desnecessários e reduzindo as ações do governo. (GRIMMELIKHUIJSEN, 2012) Para Dror (1999), a transparência é semelhante ao que os gregos chamavam de um fármaco, um material que cura apenas se tomado na dose certa.

Quando o foco é a investigação do uso de tecnologia da informação, à transparência geralmente está associada a dimensão de acessibilidade. Assim, são avaliadas questões tais como a receptividade do site à participação dos usuários, com materiais de treinamento (THORNTON; THORNTON, 2013) e ferramentas de suporte (i.e., mapa do site, ferramenta interna de busca), a capacidade de oferecer ajuda para facilitar o acesso às informações, as características que permitem ao usuário confiar no serviço prestado e facilitam o acesso a portadores de necessidades especiais e a funcionalidade estética e praticidade da distribuição das informações. (ALVES; SOUSA, 2011; MARENGO; DIEHL, 2011) Lourenço e colaboradores (2013) também incluem a visibilidade (como cada item torna-se visível no site) e o formato (ou apresentação) da informação. No que diz respeito aos "conteúdos" a serem tornados transparentes, na literatura nacional, a prestação de contas se materializa na divulgação da execução orçamentária, da gestão dos ativos e das dívidas do ente federativo de que se trate (CRUZ; ÁLVARO, 2012), nas publicações relacionadas a licitações e contratos (JACQUES; QUINTANA; MACAGNAN, 2013), assim como na divulgação do plano plurianual, da lei de diretrizes orçamentária e a lei orçamentária anual. (JACQUES; QUINTANA; MACAGNAN, 2013; MARENGO; DIEHL, 2011) Não só a publicação é suficiente, pois as atualizações, assim como os intervalos temporais da informação, também contam. Nesse sentido, Thornton e Thornton (2013) olha para o total de anos de dados históricos disponíveis, e Raupp e Pinho (2011) utilizam indicadores sobre a divulgação no prazo, parcial ou fora do prazo do conjunto de relatórios legais, assim como das suas versões simplificadas. A Associação Contas Abertas também avalia o quesito "Série Histórica e Frequência de Atualização" ao realizarem o índice 
de transparência dos estados e capitais brasileiras. Contudo, a transparência emerge como mais do que a questão contábil e financeira, pois órgãos transparentes também deveriam divulgar informações institucionais e outras mais gerais, entre elas: legislação (RAUPP; PINHO, 2011), informação sobre os representantes (CRUZ; ÁLVARO, 2012; JACQUES; QUINTANA; MACAGNAN, 2013), sobre os órgãos - estrutura, composição, funcionamento -, assim como da remuneração dos servidores e representantes. (JACQUES; QUINTANA; MACAGNAN, 2013) Finalmente, há autores que inserem o elemento interativo - ou até participativo - ao avaliarem a transparência. Raupp e Pinho (2011) e Jacques, Quintana e Macagnan (2013) olham para a existência de canais de contato (desde os mais básicos, como telefone, até os mais formais, como ouvidoria, passando por alguns mais interativos, como chats) assim como do monitoramento das ações dos usuários. Também, a possibilidade de oferecimento à população de instrumentos de participação no orçamento e em outros planos municipais.

Em síntese, a natureza ampla do conceito de transparência é bem elucidada em trechos de Jacques, Quintana e Macagnan (2013) e Cruz e Álvaro (2012), ao afirmarem que se procura prover o cidadão de informações claras e objetivas em áreas consideradas de maior interesse e cujas condições têm impacto direto na vida dos cidadãos: política tributária, administração, licitações e compras, segurança pública, educação, cultura, esporte e lazer, habitação e urbanismo, saúde, saneamento básico, gestão ambiental, atividades econômicas (indústria, comércio, serviços e agricultura), trabalho e transportes. Finalmente, a transparência também parece estar ligada aos dados abertos, pois as informações disponíveis, isto é, documentos, textos e relatórios, deveriam ser passíveis de download (RAUPP; PINHO, 2011), embora os dados abertos exijam outras características para serem considerados "abertos".

A literatura internacional vai em igual direção à nacional no que diz respeito à caracterização e à avaliação da transparência. Há transparência da atividade governamental na publicação de agendas e atas de reuniões, assim como na publicação de informação financeiras, sobre funcionários, e sobre a comunidade. (ARMSTRONG, C., 2011) 
Ao abordar as dimensões e indicadores da transparência on-line, Frick (2008) contempla a natureza ampla do conceito ao propor cinco dimensões. "Informação" referir-se-ia ao fornecimento de informações detalhadas sobre as funções do governo e dos funcionários. "Participação" constituiria a segunda dimensão, sobre a interação com os cidadãos através de ferramentas on-line para consulta e participação. "Relatório", diz respeito ao fornecimento de detalhes sobre decisões e ações de organizações do setor público, ou seja, da própria atividade pública pelos indicadores de desempenho. “Openness”, procuraria permitir que os usuários comparassem o desempenho do servidor público contra parâmetros pré-estabelecidos (arquivos dos discursos presidenciais, indicadores comparativos, indicadores sobre o plano do governo e seus objetivos específicos ao longo do tempo) e a última, "Accountability", visa permitir que os usuários adotem algum mecanismo de controle de recompensa ou punição - sobre os servidores públicos, através de mecanismos publicados para entrar em contato com os responsáveis de sites, da possibilidade de reclamar ou comentar sobre o desempenho dos empregados do governo e a presença de links de contato ou informações com o Provedor de Justiça.

Por fim, faz-se necessário estabelecer o processo que ligar Transparência à Accountability. Se considerarmos a existência de informações de fácil acesso e compreensão por parte dos cidadãos, e que estes, de posse delas, podem avaliar determinada situação ou processo decisório. Presume-se, também, que depois de ter analisado tais informações, esses cidadãos possam cobrar justificativas para os atos das autoridades públicas (responsabilidade) e, caso não se satisfaçam, prestarem queixa às instituições formais de controle em relação a algo que tenham identificado como desvio do padrão de comportamento esperado por parte do agente público.

Desse modo, tão importante quanto tornar disponível a informação de fácil compreensão para os cidadãos (dar transparência) é disponibilizar para a sociedade acesso às instituições de controle (accountability) para que o ciclo entre ambos se complete e, de fato, promova avanços substantivos da democracia ao possibilitar aos governados cobrar responsabilidade de seus governantes. 


\section{REFERÊNCIAS}

ACEMOGLU, D.; JOHNSON, S.; ROBINSON, J. A. Reversal of fortune: geography and institutions in the making of the modern world income distribution. Quarterly journal of Economics, v. 118, n. 4, p. 1231-1294, Nov. 2002.

AIKINS, S. K.; KRANE, D. Are public officials obstacles to citizencentered e- government? An examination of municipal administrators' motivations and actions. State and Local Government Review, v. 42, n. 2, p. 87-103, Aug. 2012.

AKUTSU, L.; PINHO, J. A. G. de. Sociedade da informação, accountability e democracia delegativa: investigação em portais de governo no Brasil. Revista de Administração Pública, Rio de Janeiro, ano 36, n. 5, p. 723-745, set./out. 2000.

ALVES, T. R.; SOUZA, C. A. Compras eletrônicas governamentais: uma avaliação dos sites de e-procurement dos governos estaduais brasileiros. Revista Eletrônica de Sistemas de Informação, Curitiba, v. 10, n. 1, jan./ jun. 2011.

ANGÉLICO, F.; ANTONIO, M.; TEIXEIRA, C. Acesso à informação e ação comunicativa: novo trunfo para a gestão social. Desenvolvimento em Questão, Ijuí, v. 10, n. 21, p. 7-27, 2012.

ARMSTRONG, C. L. Providing a clearer view: An examination of transparency on local government websites. Government Information Quarterly, v. 28, n. 1, p. 11-16, Jan. 2011.

ARMSTRONG, E. Integrity, transparency and accountability in public administration: recent trends, regional and international developments and emerging issues. New York: United Nations, 2005.

BAIRRAL, M. A. da C.; SILVA, A. Transparência no setor público: uma análise do nível de transparência dos relatórios de gestão dos entes públicos federais no exercício de 2010. In: ENCONTRO DA ASSOCIAÇÃO NACIONAL DE PÓS-GRADUAÇÃO E PESQUISA EM ADMINISTRAÇÃO, Rio de Janeiro, 2013. Anais... Rio de Janeiro, 2013. p. 1-16.

BEHN, R. D. Rethinking democratic accountability. Washington, DC: Brookings Institution Press, 2001. 
BERTOT, J. C.; JAEGER, P. T.; GRIMES, J. M. Promoting transparency and accountability through ICTs, social media, and collaborative e-government. Transforming Government: People, Process and Policy, v. 6, n. 1, p. 78-91, 2012.

BERTOT, J. C.; JAEGER, P. T.; GRIMES, J. M. Using ICTs to create a culture of transparency: E-government and social media as openness and anti-corruption tools for societies. Government Information Quarterly, v. 27, n. 3, p. 264-271, July, 2010.

BOBBIO, N. O futuro da democracia. Rio de Janeiro: Paz e Terra, 2000.

BOVENS, M. A. P. Analysing and Assessing Accountability: A Conceptual Framework. European Law Journal, v. 13, n. 4, p. 447-468, July, 2007.

CAMPOS, A. M. Accountability: quando poderemos traduzi-la para o português? Revista de Administração Pública, Rio de Janeiro, v. 24, n. 2, p. 30-50, fev./abr. 1990.

CENEVIVA, R.; FARAH, M. R. S. Avaliação, informação e responsabilização no setor público. Revista de Administração Pública, Rio de Janeiro, v. 46, n. 4, p. 993-1016, jul./ago. 2012.

CERRILLO-I-MARTÍNEZ, A. The regulation of diffusion of public sector information via electronic means: Lessons from the Spanish regulation. Government Information Quarterly, v. 28, n. 2, p. 188-199, Apr. 2011.

CHEN, Y.-C. A comparative study of e-government XBRL implementations: The potential of improving information transparency and efficiency. Government Information Quarterly, v. 29, n. 4, p. 553-563, Oct. 2012.

CRUZ, C. F. et al. Transparência da gestão pública municipal: um estudo a partir dos portais eletrônicos dos maiores municípios brasileiros. Revista de Administração Pública, Rio de Janeiro, v. 46, n. 1, p. 153-176, jan./fev. 2012.

CRUZ, C. F.; SILVA, L. M.; SANTOS, R. Transparência da gestão fiscal: um estudo a partir dos portais eletrônicos dos maiores municípios do Estado do Rio de Janeiro. Contabilidade, Gestão e Governança, Rio de Janeiro, v. 12, n. 3, p. 102-115, 2009. 
CULLIER, D.; PIOTROWSKI, S. J. Internet information-seeking and its relation to support for access to government records. Government Information Quarterly, v. 26, n. 3, p. 441-449, July, 2009.

DAWES, S. S. Stewardship and usefulness: Policy principles for information-based transparency. Government Information Quarterly, v. 27, n. 4 , p. 377-383, Oct. 2010.

DEL SOL, D. A. The institutional, economic and social determinants of local government transparency. Journal of Economic Policy Reform, v. 16, n. 1, p. 90-107, Jan. 2013.

DROR, Y. Transparency and openness of quality democracy. In: NISPACEE CIVIL SERVICE FORUM, 2., 1999. Maastricht, Netherlands.

Proceedings... Maastricht, Netherlands: EIPA, 1999. Disponível em: <http://www.nispa.org/files/publications/ebooks/nispaceeopennes2000.pdf\#page $=64>$. Acesso em: 10 nov. 2014 .

FLICK, M. Political mediation of e-Transparency. International journal of Electronic Government Research, v. 4, n. 3, p. 81-102, 2008.

FONTES FILHO, J. R.; NAVES, G. G. A contribuição do Sistema Integrado de Administração Financeira do Governo Federal (SIAFI) para a promoção da accountability horizontal: a percepção dos usuários. Brazilian Business Review, v. 11, n. 3, p. 1-28, maio/jun. 2014.

FRANCO, L. M. G et al. Nível de divulgação eletrônica da contabilidade pública dos municípios do Paraná no ambiente da internet. Revista de Ciências da Administração, v. 16, n. 38, p. 140-153, abr. 2014.

GRIMMELIKHUIJSEN, S. G. A good man but a bad wizard. About the limits and future of transparency of democratic governments. Information Polity, v. 17, p. 293-302, July, 2012.

GRIMMELIKHUIJSEN, S. G. Do transparent government agencies strengthen trust? Information Polity, v. 14, n. 3, p. 173-186, Aug. 2009.

GRIMMELIKHUIJSEN, S. G.; WELCH, E. W. Developing and Testing a Th eoretical Framework for Computer-Mediated Transparency of Local Governments. Public Administration Review, v. 72, n. 4, p. 562-571, Jul./ Aug. 2012. 
GRIMMELIKHUIJSEN, S. et al. The Effect of Transparency on Trust in Government: A Cross-National Comparative Experiment. Public Administration Review, v. 73, n. 4, p. 575-586, Jul./Aug. 2013.

HALACHMI, A.; GREILING, D. Transparency, E-Government, and Accountability. Public Performance \& Management Review, v. 36, n. 4, p. 572-584, Dec. 2013.

HOLMSTRÖM, B. Moral hazard and observability. The Bell Journal of Economics, v. 10, n. 1, p. 74-91, Spring, 1979.

HOOD, C. Transparency in a historical perspective. In: C. HOOD; D. HEALD (Ed.). Transparency: The key to better governance. Oxford: Oxford University Press, 2006. p. 3-23.

JACQUES, F. V. S; QUINTANA, A. C.; MACAGNAN, C. B.

Transparência em municípios da Região Sul do Brasil. In: ENCONTRO DA ASSOCIAÇÃO NACIONAL DE PÓS-GRADUAÇÃO E PESQUISA EM ADMINISTRAÇÃO, Rio de Janeiro, 2013. Anais... Rio de Janeiro, 2013. p. 1-13.

KAUFMANN, D.; KRAAY, A. Growth without governance. 2002. (World Bank Policy Research Working, 2928)

LIEM, S. I. Constituents of transparency in public administration with reference to empirical findings from Estonia. $320 \mathrm{f} .2007$. Dissertation (Doctor of Economics) - University of St. Gallen, St. Gallen, 2007. Disponível em: <http://verdi.unisg.ch/www/edis.nsf/ wwwDisplayIdentifier/3350/\$FILE/dis3350.pdf>. Acesso em: 10 nov. 2014 .

LOUREIRO, M. R.; TEIXEIRA, M. A. C; PRADO. Construção de instituições democráticas no Brasil contemporâneo: transparência das contas públicas. Organização Q Sociedade, Salvador, v. 15, n. 47, p. 107119, out./dez. 2008.

LOURENÇO, R. P. et al. Online Transparency for Accountability: One Assessing Model and two Applications. The Electronic Journal of e-Government, v. 11, n. 2, p. 280-292, 2013.

LUHMANN, N. Familiarity, Confidence, Trust: Problems and Alternatives, In: GAMBETTA, D. (Ed.). Trust: Making and Breaking 
Cooperative Relations. Oxford: Department of Sociology, University of Oxford, 2000. Cap. 6, p. 94-107.

MARENGO, S. T.; DIEHL, C. A. A possibilidade do controle social mediante o acesso a informações em sites dos municípios gaúchos. Contabilidade, Gestão e Governança, Brasília, v. 14, n. 3, p. 120-133, set./ dez. 2011.

MEDEIROS, A. K.; CRANTSCHANINOV, T. I.; SILVA, F. C. Estudos sobre accountability no Brasil: meta-análise de periódicos brasileiros das áreas de administração , administração pública, ciência política e ciências sociais. Revista de Administração Pública, Rio de Janeiro, v. 47, n. 3, p. 745-775, maio/jun. 2013.

MEIJER, A. J. Understanding Computer-Mediated Transparency. International Review of Administrative Sciences, v. 75, n. 2, p. 255-269, 2009.

MEIJER, A. J. Publishing performance results on the Internet: Do stakeholders use the Internet to hold Dutch public service organizations to account? Government Information Quarterly, v. 24, n.1, p. 165-185, Jan. 2007.

MEIJER, A. J. Transparent government: parliamentary and legal accountability in an information age. Information Polity, v. 8, n. 1-2, p. $67-78,2003$.

MEIJER, A. J. Being transparent or spinning the message? An experiment into the effects of varying message content on trust in government. Information Polity, v. 16, p. 35-50, 2011.

MEIJER, A. J., BANNISTER, F., GRIMMELIKHUIJSEN, S. Being transparent or spinning the message? An experiment into the effects of varying message content on trust in government. Information Polity, v. 16, n. 1, p. 35-50, Jan. 2011.

MICHENER, G.; BERSCH, K. Identifying transparency. Information Polity, v. 18, p. 233-242, 2013.

MOON, M. J. The evolution of e-Government among municipalities: Rhetoric or reality. Public Administration Review, v. 62, n. 4, p. 424-433, July/Aug. 2002.

MULGAN, R. Truth in government and the politicization of public service advice. Public Administration, v. 85, n. 3, p. 569-586, Sept. 2007. 
PARAJULI, J. A Content Analysis of Selected Government Web Sites: a Case Study of Nepal. The Electronic Journal of e-Government, v. 5, n. 1, p. 87-94, 2007.

PINHO, J. A. G. Investigando portais de governo eletrônico de estados no Brasil: muita tecnologia, pouca democracia. Revista de Administração Pública, Rio de Janeiro, v. 42, n. 3, p. 471-493, maio/jun., 2008.

PINHO, J. A. G.; SACRAMENTO, A. R. S. Accountability: já podemos traduzi-la para o português? Revista de Administração Pública, Rio de Janeiro, v. 43, n. 6, p. 1343-1368, nov./dez., 2009.

QUINN, A. C. Keeping the citizenry informed: Early congressional printing and 21 st century information policy. Government Information Quarterly, v. 20, n. 3, p. 281-293, July, 2003.

RAUPP, F. M.; PINHO, J. A. G. de. Construindo a accountability em portais eletrônicos de câmaras municipais: um estudo de caso em Santa Catarina. Cadernos EBAPE, Rio de Janeiro, v. 9, n. 1, p. 117-139, mar. 2011.

RELLY, J. E.; SABHARWAL, M. Perceptions of transparency of government policymaking: A cross-national study. Government Information Quarterly, v. 26, n. 1, p. 148-157, Jan. 2009.

REYLEA, H. C. Federal freedom of information policy: Highlights of recent developments. Government Information Quarterly, v. 26, n. 2, p. 314-320, Apr. 2009.

RIBEIRO, M. M; MATHEUS, R; VAZ, J. C. New perspectives for electronic government: the adoption of open government data in brazil. In: INTERNATIONAL CONFERENCE ON INFORMATION SYSTEMS AND TECHNOLOGY MANAGEMENT, São Paulo, 2011. Anais... São Paulo, 2011.

ROCHA, A. C. A realização da accountability em pareceres prévios do Tribunal de Contas de Santa Catarina. Revista de Administração Pública, Rio de Janeiro, v. 47, n. 4, p. 901-926, jul./ago. 2013.

ROCHA, A. C. accountability na administração pública: modelos teóricos e abordagens. Contabilidade, Gestão e Governança, Brasília, v. 14 , n. 2, p. $82-97$, set. 2011.

SHULER, J. A.; JAEGER, P. T.; BERTOT, J. C. Implications of harmonizing e- government principles and the Federal Depository 
Library Program (FDLP). Government Information Quarterly, v. 27, n. 1, p. 9-16, Jan. 2010.

THORNTON, J. B.; THORNTON, E. Assessing state government financial transparency websites. Reference Services Review, v. 41, n. 2 , p. 366-387, 2013.

TOLBERT, C. J.; MOSSBERGER, K. The effects of e-government on trust and and confidence in government. Public Administration Review, v. 66, n. 3, p. 354-369, May/June, 2006.

WELCH, E. W. The relationship between transparent and participative government: A study of local governments in the United States. International Review of Administrative Sciences, v. 78, n. 1, p. 93-115, Mar. 2012.

WILLIAMSON, O. E. The new institutional economics: taking stock; looking ahead. Journal of Economic Literature, v. 38, p. 595-613, Sept. 2000. 